\title{
Experiencia española de transición: desde la dispersa normativa de la edificación prescriptiva hacia el nuevo Código Técnico de la Edificación 2006, una norma moderna y unificada, enfocada hacia las prestaciones ${ }^{1}$
}

\author{
Spanish Transition Experience: From Scattered Prescriptive Building Regulations to the New \\ 2006 Technical Building Code, a Modern and Unified Norm, Focused on Performance
}

\author{
Javier Serra ${ }^{(1)}$, JoséAntonio Tenorio ${ }^{(2)}$ \\ (1) Arquitecto, Universidad Politécnica de Madrid.Coordinador de la Unidad de Edificación Sostenible del Ministerio de Fomento del \\ Gobierno de España. jserra@fomento.es \\ (2) Ingeniero de Caminos, Canales y Puertos, Universidad Politécnica de Madrid.Coordinador de la Unidad de Calidad en la Construcción \\ del Instituto de Ciencias de la Construcción Eduardo Torroja, CSIC en Madrid, España. tenorio@ietcc.csic.es
}

Recibido 8 de septiembre de 2014. Modificado 23 de enero de 2015. Aprobado 25 de enero de 2015.

DOI: http://dx.doi.org/10.16924/riua.v0i41.788

\author{
Palabras clave \\ Código basado en prestaciones, código nórdico, construc- \\ ción, España.
}

\section{Resumen}

El Código Técnico de la Edificación español se ha inspirado en las experiencias de los países que han adoptado el denominado enfoque prestacional ("Performance-Based Building Codes”). Los códigos prestacionales pueden favorecer la adopción de soluciones innovadoras que no encuentran lugar en el enfoque prescriptivo, rígido y cerrado a la innovación. Es importante que el proceso de elaboración del código sea participativo, y en cierto modo 'bottom-up', como ha sido el caso español, y muy apoyado por el conocimiento y la experiencia del mundo académico, científico y de investigación. Es necesaria también la existencia de mecanismos para asegurar su permanente puesta al día, de forma que el código esté atento a la evolución tecnológica y a las demandas sociales.

\section{Key words}

Performance-Based Building Codes, construction, nordic code, Spain.

\begin{abstract}
The Spanish Technical Building Code has been inspired by the experiences of countries that have adopted the socalled Performance-Based Building Codes. These codes may advance the use of innovative solutions that do not fit within a prescriptive, rigid and closed approach to innovation. It is important that the code be developed in a participatory and, to some extent, bottom-up way, as has been the case in Spain, and heavily supported by knowledge and experience from the worlds of academia, science and research. Mechanisms must also be in place to ensure that it is kept up to date, so that the code responds to technological developments and social demands.
\end{abstract}

\section{INTRODUCCIÓN}

En el 2006 fue aprobado en España un nuevo código de la edificación que superaba un régimen anterior normativo, iniciado en los años sesenta con las primeras normas de tipo estructural, de acciones en edificaciones y de protección contra el sismo. Estas normas habían surgido en el último tercio del siglo pasado cumpliendo entonces su función, pero a medida que pasaba

${ }^{1}$ Este artículo es el resultado de la ponencia presentada por Javier Serra y José Antonio Tenorio en el foro "Los códigos en la construcción en Colombia: Aproximaciones y Aplicaciones", realizado en la Universidad de los Andes el 8 de septiembre de 2014. 


\section{Prestaciones (desempeños)}

\section{Lo prescriptivo frente a lo prestacional Conceptos}

\section{Pensar en términos de fines y no de medios}

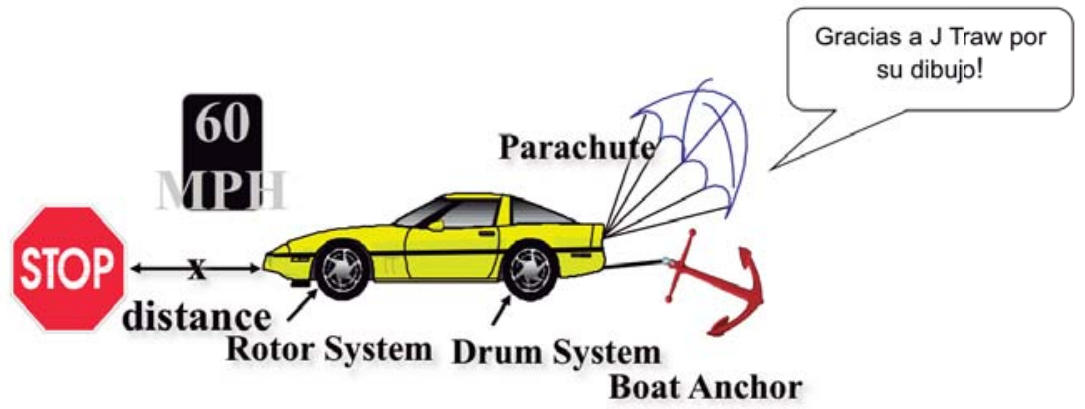

Figura 1. Descripción del enfoque prestacional. Fuente. Elaboración propia

el tiempo y estas normas necesitaban actualizarse, se apreció una cierta insatisfacción debido a que estas eran prescriptivas y no facilitaban a los agentes -industria, proyectistas, constructores, entre otros - la adopción, las innovaciones y los desarrollos nuevos. Había llegado el momento de cambiar de enfoque y comenzar a pensar en términos de fines y no de medios, por lo que el enfoque prestacional que iban adoptando otros países nos parecía el más adecuado.

Este enfoque prestacional queda descrito claramente en el siguiente gráfico (Figura 1) que tomamos prestado de Jon Traw, responsable del código americano International Building Code (IBC) elaborado por la International Conference of Building Officials (ICBO) de los EEUU, ahora integrada en el International Code Council (ICC), con quien hemos colaborado en el Comité Internacional de Colaboración de Códigos (IRCC), el cual expresa que a un automóvil se le pueden pedir requisitos reglamentarios de dos maneras: de una manera prescriptiva, como que el auto debe adoptar frenos de disco de determinado diámetro o fuerza; o, se le puede pedir que sea capaz de realizar una frenada a una cierta distancia y velocidad, dejando al fabricante de automóviles la libertad para que busque el método más adecuado.

Sin embargo, el concepto de las prestaciones en los edificios no es nuevo y empieza a aparecer en la construcción en la década de los setenta en Europa a través de la UEAtc (Unión Europea para la Aprobación Técnica en la Construcción) que trata de promover y facilitar la incorporación de productos innovadores en la construcción, como también lo hace el HUD (Departamento de Vivienda y Desarrollo Urbano de los EEUU) desde los años sesenta tratando de promover este enfoque en el diseño y construcción de casas, eliminando así las barreras técnicas de las normas prescriptivas.

El interés por las prestaciones (desempeños) está apoyado por el mundo científico, por la industria, la normalización y también por la reglamentación no solo nacional sino la de carácter internacional. En el ámbito de la colaboración científica en la edificación tenemos al Consejo Internacional de Edificación (CIB) creado en 1953 tras la postguerra europea al servicio de la comunidad de la construcción y de la edificación. El CIB ha venido trabajando en estas iniciativas a través de grupos de trabajo como el TG11 (1995-1997) y TG37 (1998-2004) en la década de los noventa. España participó en estos grupos compartiendo experiencias con otros países pioneros, lo que nos sirvió de base para nuestro cambio de modelo. Es destacable el documento final del TG11 (CBI, 1997), en gran medida inspirador de nuestro cambio normativo. Posteriormente, el ya citado IRCC, creado en 1996, tomó el relevo de estos grupos de trabajo del CIB y también España se incorporó en él en 1999. Se puede apreciar que entre los miembros del IRCC hay países de Asia, de América y de Europa.

Por otro lado, hablando ya del mundo reglamentario, tenemos que referirnos necesariamente como hito histórico a la experiencia escandinava surgida en los años setenta entre los propios países del norte de Europa que teniendo culturas, sistemas constructivos y lenguas parecidas, tenían mercados herméticos donde no podían importarse o exportarse productos de construcción e incluso casas hechas en un país u otro por culpa de sus reglamentos técnicos. De allí que desde el comité nórdico NKB se planteara preparar una armonización entre ellos que posteriormente y, por instancia de aquellos, también tuvo lugar en el Comité Económico para Europa, CEPE, de Naciones Unidas, asimilado en la legislación de la propia Unión Europea y también en la propia Organización Mundial del Comercio OMC.

El Comité Nórdico de Edificación $\mathrm{NKB}$, con el fin de armonizar las normas discordantes de la edificación de sus miembros, como se ha dicho, fue inspirador de la famosa jerarquía de cinco niveles (Figura 2). De este esquema aprendemos que cualquier norma o código de la construcción debe basarse en unos 'principios' que estarán en la cúspide de este triángulo y corresponden a los objetivos básicos de la sociedad; continúa con el establecimiento de unos requisitos funcionales o prestacionales que a su vez deben apoyarse en unos métodos de verificación y en un conjunto de soluciones aceptables que 
en muchos casos eran las que precisamente habían sido adoptadas en los códigos prescriptivos.

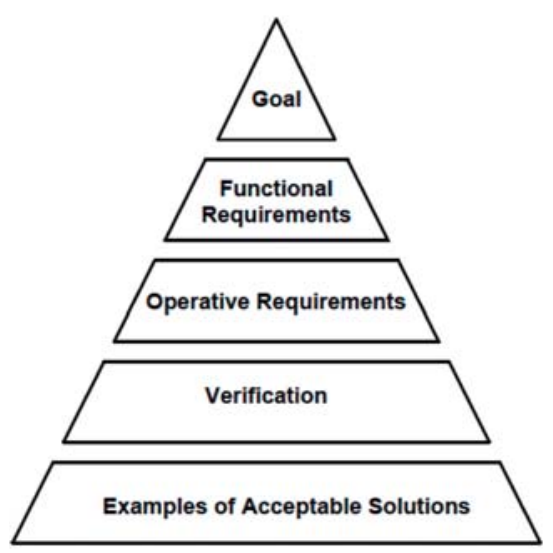

NKB Model (NKB, 1976)

Figura 2. Jerarquía del Nordic Committee on Building Regulations (NKB). Fuente. NBK, 1978.

Es importante mencionar que la Organización Mundial del Comercio (OMC) también se ha sumado a este movimiento en los años 2000. Son numerosos los países que adoptan acuerdos sobre supresión de obstáculos técnicos al comercio que contemplan la obligación de que sus miembros especifiquen en sus reglamentos técnicos las exigencias en forma prestacional, de modo que el intercambio de mercancías y productos entre los países de la OMC no se vea afectado por barreras técnicas.

Como se citaba antes, en el seno de la Comisión Económica Para Europa (CEPE) de las Naciones Unidas existió un ámbito de colaboración muy interesante que en la década de los ochenta dio lugar a un "Compendio de disposiciones modelo de reglamentos de Construcción”, que fue la base después para lo que en la Unión Europea vinieron a desarrollar las directivas europeas basadas en lo que se llamó el 'nuevo enfoque' que era precisamente el enfoque prestacional.

Esto sucedió en Europa en los años ochenta, donde a pesar de los Tratados y del Mercado Común no existía un mercado interior único pues la armonización técnica y reglamentaria en
Europa para muchísimas mercancías y productos, incluidos los de la construcción, no avanzaba. Por ello fue necesario el entonces denominado 'nuevo enfoque’ de 1985 que dio lugar a un conjunto de directivas entre las que destacamos la 89/106/CE de productos de construcción aprobada en 1989, en donde quedaron establecidos estos mismos principios, que habrían de ser adoptados obligatoriamente por los estados miembros.

La normalización europea elaborada por organismos como el Comité Europeo de Normalización (CEN), el Comité Europeo de Normalización Electrotécnica (CENELEC) y el Instituto Europeo de Normas de Telecomunicaciones (ETSI) es la base de estas directivas europeas de la misma manera que lo son las normas ISO en la esfera internacional para la OMC. De esta forma, se cuenta con un conjunto de estándares de apoyo para las normativas y códigos que son muy importantes y que sirven para referirse en ellos sin tener que bajar a los detalles técnicos que hablan de los productos, de sus especificaciones y de sus pruebas y sus ensayos.

En España toda esa transformación ha venido surgiendo en el cambio de milenio. Así, en 1999 se aprueba una nueva ley que el sector venía reclamando desde hacía décadas: la Ley de la Ordenación de la Edificación LOE, que es el nuevo marco para la calidad de la edificación. Un marco que establece, no solo la definición de los requisitos básicos que han de cumplir los edificios, sino también establece un marco ordenador de los agentes de la edificación, que enumera otorgando a cada uno de ellos unas determinadas competencias, obligaciones y responsabilidades. Además, establece un mecanismo de garantías mediante un seguro anual para los defectos, mala construcción; otro trienal para los de habitabilidad y un tercero decenal de daños referidos a los aspectos estructurales. La Ley de la Edificación a la vez obliga a que el Gobierno prepare y apruebe un nuevo marco técnico que de por superada la pléyade, el conjunto de normas básicas de la edificación que se había venido preparando en las décadas anteriores, haciendo que el código sea el mejor instrumento, basado en las prestaciones, para alcanzar los objetivos finales de mejorar la calidad de la edificación.

\section{El NUEVo CÓDIGo TÉCNICO DE LA EDIFICACIÓN}

El código técnico de la edificación español desarrolla los requisitos básicos que enuncia la Ley de Ordenación de la edificación que se agrupan en torno a tres paquetes.

El primer grupo es el relativo a la funcionalidad, utilización, accesibilidad y acceso a los servicios de telecomunicación. El primero de ellos no se contempla en el código dado que son requisitos de tipo espacial que ya se regulan en las ordenanzas municipales o en las normas regionales que hay en España. Hay que tener en cuenta que existen en España 17 comunidades autónomas que tienen libertad para regular esta materia. Aunque la accesibilidad se ha incorporado al código recientemente, la normativa de telecomunicación es independiente del código por ser competencia de otro departamento ministerial, si bien está coordinada con él.

El segundo paquete trata todo lo relativo a los aspectos de seguridad, la estructural en primer término y que es lo que primariamente han desarrollado los códigos; a continuación la seguridad en caso de incendio, y en tercer lugar, la seguridad de utilización (que recientemente se ha unido con la accesibilidad), de modo que el edificio en su uso normal no suponga riesgo de accidente para las personas en aspectos tales como caída, resbalamiento, quemaduras, etc. Por último, están los requisitos relativos a lo que hemos llamado habitabilidad que compendian la higiene, la salud y la protección del medio ambiente que lo hemos resumido en el término salubridad, la protección contra el ruido, y finalmente, lo que en mayor medida preocupa en este momento a toda la Unión Europea, el ahorro 
de energía y aislamiento térmico. De modo que esta es la estructura que tiene el código técnico español.

La preparación del código, finalmente aprobado en el 2006, se inició en 2002 y es un trabajo que no tiene fin. De forma permanente, y utilizando para ello las tecnologías de la información como la página web (www.codigotecnico.org), se trabaja día a día en un sistema de realimentación con los miles de usuarios del código que mandan sugerencias y hacen preguntas, de forma que el código desde el 2006 ha sido puesto al día y actualizado en numerosas ocasiones.

A la hora de analizar la normativa de construcción de un país lo primero en lo que se debe pensar es en que esté acorde con los intereses de la sociedad y en que se interprete desde los departamentos del gobierno competentes. Cada vez que reglamentariamente se eleva el nivel de prestación de un edificio, se produce un impacto en el coste del edificio, y por lo tanto a la hora de establecer los niveles de prestaciones de los diferentes requisitos, dejando de lado los de seguridad que han de ser más que suficientes, como los acústicos o los energéticos, se han de tener en cuenta las necesidades reales del país y de cómo puede ser abordable ese impacto para poder hacerlo acorde con las necesidades reales y actuales, de forma que la regulación sea eficaz, desde el punto de vista de coste-beneficio.

En 2000 nada más aprobarse la LOE, el Ministerio de Fomento redactó un estudio de bases que orientara sobre cómo debería ser el nuevo código técnico y se encargó al Instituto Torroja para que empezara a trabajar con el Ministerio, dándole apoyo técnico, y realizara una investigación aplicada. Fue un trabajo conjunto interesante y realizado bajo la dirección del Ministerio, en el que participaron el Instituto Torroja, y otras instituciones del mundo académico. Como se ha dicho, se partía de la experiencia internacional y del modelo de códigos técnicos prestacionales que se ha descrito antes. Todos consideraban que introducir la filosofía de la prestación en los códigos era un reto valioso que se podía poner en marcha y que se podía trabajar en su desarrollo.

Lo importante era involucrar a todos los agentes de la edificación, de una menor o mayor manera permitiéndoles participar en la gestación de ese nuevo código que superase la antigua, obsoleta, sobreabundante y a la vez incompleta, normativa existente para conseguir una normativa actualizada. Después de una década de trabajo, felizmente se cuenta en España con un código lo suficientemente avanzado, basado en lo posible en prestaciones, pero como se ha dicho antes este es un trabajo que nunca se termina. Habrá que seguir trabajando sobre él y habrá que seguir avanzando.

Antes de hablar de prestaciones es importante señalar que para avanzar en la tecnología de la edificación y permitir que las innovaciones puedan surgir y puedan tener cabida en la construcción, como en cualquier otro campo, hace falta investigación, que en gran parte viene desde el mundo académico. No vale adoptar soluciones de otros países o de otras culturas, es necesario avanzar en la generación de soluciones propias que provengan del mundo constructivo, y hacerlas soluciones propias. Es muy importante por tanto contar con la investigación como apoyo a la normativa, que conferirá a los proyectistas y a los ciudadanos la seguridad de que no haya problemas estructurales, como derrumbes o caídas, de que se garantice la habitabilidad del edificio. De forma que en sí lo que va a hacer el reglamento es fijar unos niveles de prestaciones, un nivel mínimo por el cual el proyecto tiene que alcanzar unos estándares de calidad y establecer unos métodos de verificación, a veces acompañados de soluciones juzgadas como satisfactorias.

\section{LA PRESTACIÓN}

La prestación se puede definir como el conjunto de características que debe tener un edificio que le hacen apto para el uso previsto, para lo que se ha diseñado, es decir, son condiciones que proporciona el edificio. No parece, por otro lado, razonable que el edificio supere en exceso algunas prestaciones pues lo único que se conseguiría es desperdiciar innecesariamente recursos económicos. A la hora de elegir un enfoque para un nuevo código técnico, hay que fijar unas bases de lo que uno quiere, hay que dar flexibilidad al sistema. Dicen algunos que la mejor reglamentación es la que no existe, que el buen proyectista tiene capacidad suficiente para saber distinguir entre lo que está bien y lo qué está mal, pero la realidad es que hay niveles que han de ser fijados obligatoriamente para defender a la sociedad.

Un código que describe exhaustivamente lo que hay que hacer, sin embargo, es un código que no permite innovar, no deja hacer las cosas de manera distinta a la que está escrita. Si se prescribe en el código, como se decía en el ejemplo del automóvil al principio, que debe tener frenos de zapatas, nunca se implementaría otro tipo de freno, como el de disco. Eso es lo que no se debe hacer en reglamentación. El asunto no es tan fácil a la hora de verificar el cumplimiento, porque en los códigos prescriptivos es más fácil comprobar que se han hecho las cosas como el código establece. Un código basado en prestaciones tiene la ventaja fundamental de que se establecen los objetivos que deben cubrirse y si el promotor, el que va a realizar el edificio, quiere unos niveles de prestación mejores sabrá poder prescribirlos también.

\section{ESTRUCTURA DEL CÓDIGO TÉCNICO ESPAÑOL}

La estructura del código técnico español, como se ha dicho antes, se basa en el código nórdico. El sistema nórdico de cinco niveles supone el establecer encima de toda la pirámide, unos objetivos prestacionales que se deben alcanzar. Si un edificio consiguiera alcanzarlos cumpliría el código técnico, pero demostrar los objetivos requiere disponer de herramientas 


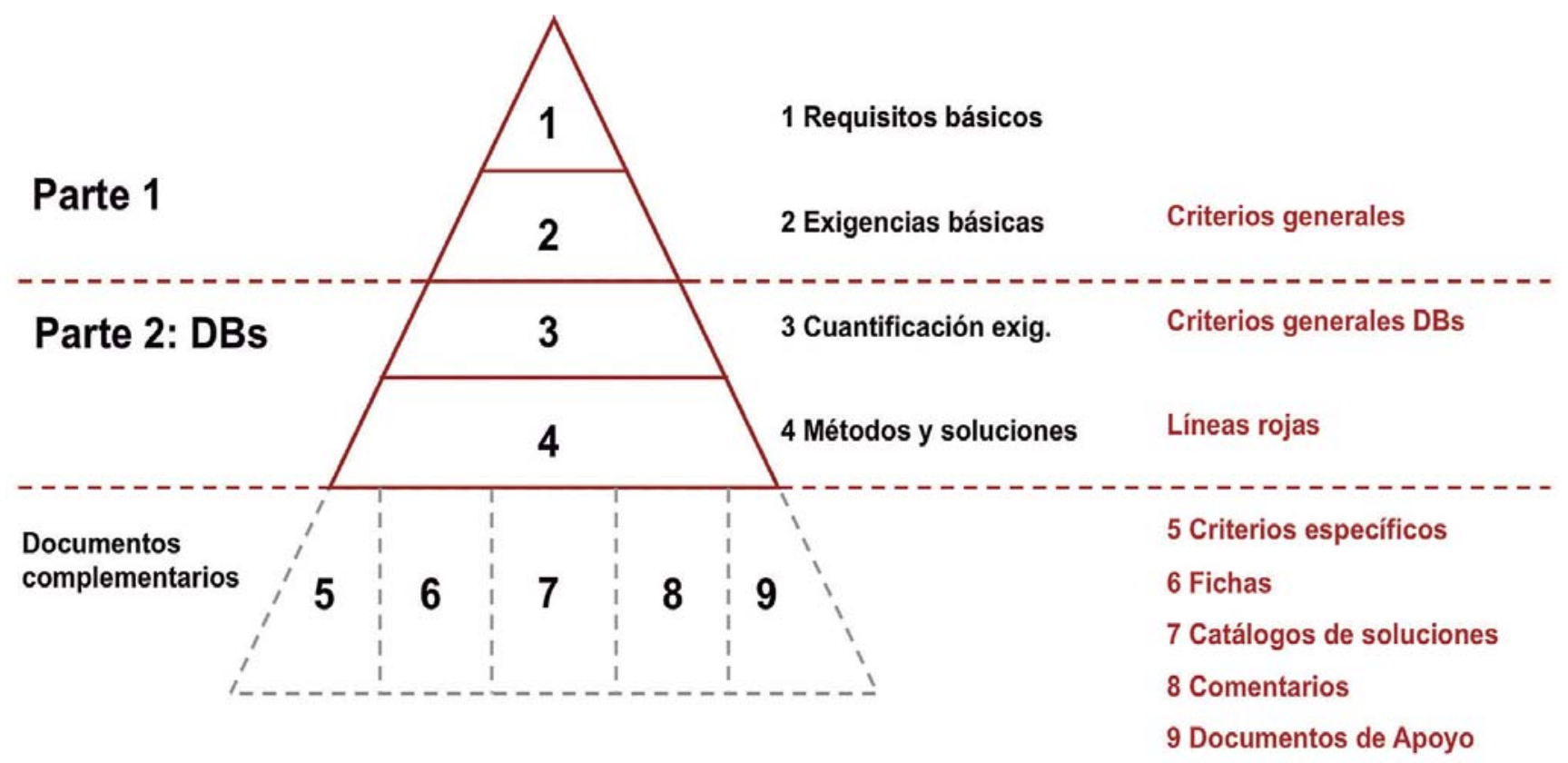

Figura 3. Estructura del código español. Fuente. Elaboración propia.

de verificación que el código también debe proporcionar. Así pues, en España el código tiene objetivos en primer lugar, que son los requisitos básicos de la LOE; a continuación se establecen exigencias técnicas, que lo que hacen es describir el nivel de prestación pero de una forma no totalmente definida. Esas exigencias se caracterizan y cuantifican dentro del propio documento hasta donde se ha podido llegar en el actual estado de conocimientos. A partir de ahí el código español ha establecido los llamados Documentos Básicos organizados por familias (Figura 3).

Sobre la base del esquema nórdico, estamos dejando en el código exclusivamente la esencia y llevamos a unos documentos que denominamos de apoyo las herramientas complementarias. En el portal (www.codigotecnico.org) se dispone libremente de toda la información referente al código español. Todos los documentos se pueden descargar de forma totalmente gratuita e incluyen desde la propia Ley de Ordenación de la Edificación hasta el último y más reciente de los documentos de apoyo, por supuesto en nuestro idioma común: español. Además hay otros documentos que son complementarios y una serie de aplicaciones informáticas que se han ido generando para facilitar el uso del código. Por ejemplo, el programa unificado Líder-Calener que permite verificar las exigencias energéticas del código y a la vez realizar la calificación energética necesaria para la obligatoria certificación energética de los edificios cuando se proyectan, venden o alquilan. Las herramientas que se han ido generando también son de libre uso y difusión. Disponemos también de una herramienta de verificación de las exigencias de protección frente al ruido que incluso es multiplataforma. Hay por ejemplo un catálogo de elementos constructivos en versión informática y en versión en papel donde se muestran soluciones constructivas de cómo se hace nuestra práctica adecuada a nuestro clima y forma de construir, que no tiene por qué coincidir con la de otros países.

Por último, mirando al futuro y sabiendo que muchas veces cuando se hacen edificios en un país o en otro el proyectista no tiene porqué pertenecer a ese país, estamos aproximando nuestro código a las normas internacionales más reconocidas. En Europa existe desde hace años un programa completo de normas sobre la seguridad estructural denominadas Eurocódigos que están empezando a ser el lenguaje común de muchos países incluso no europeos. En Singapur por ejemplo ya los están utilizando. Son normas voluntarias pero sus métodos de cálculo están avalados por los máximos especialistas y la experiencia.

Para concluir, quisiéramos compartir con el mundo científico y académico hispanoparlante, ahora y en el futuro nuestra experiencia en el desarrollo de códigos técnicos.

\section{REFERENCIAS}

Becker, R. \& Hattis, D. B. (1999). Comparison of the systems approach and the nordic model and their melded application in the development of performance based building codes and standards. Israel, USA. Recuperado dehttp://cibworld.xs4all. nl/pebbu_dl/resources/literature/ downloads/01General/06HattisBecker.pdf 
Bjorkmann, W. R. (2009, junio- julio). Experience in introducing performance based fire regulations. Ponencia presentada en 8th International Fire Science \& Engineering Conference, Edinburgh, Scotland.

MinisteriodeFomentodeEspaña(2010). Código Técnico de la Edificación. Madrid, España. Recuperado de http://www.codigotecnico.org/web/

Nordic Committee on Building Regulations (NKB). (1978). Structure for Building Regulations, Report No. 34, Stockholm, Sweden.
Oleszkiewicz, I., National Research Council Canada (NRCC), Institute for Research in Construction. (1997). Final Report of CIB Task Group 11 Performance-Based Building Codes. Canada. Recuperado de http://cibworld.xs4all.nl/dl/ ib/9801/pages/9801204.html

Performance based building thematic network. Website. Rotterdam, The Netherlands. Recuperado de http:// www.pebbu.nl/

Spekkink, D. (2005). Performance based design of buildings. The
Netherlands. Recuperado de http:// www.pebbu.nl/resources/allreports/ downloads/07_D3_FinalReport.pdf

United Nations Development Group. (2014). Website. Recuperado de http://www.undg.org/docs/13180/ UN-Guidelines-masterV1.pdf

Wijkström, E. \& McDaniels, D. (2013). International standards and the WTO TBT Agreement: Improving governance for regulatory alignment. Geneva, Switzerald. Recuperado de http://www.wto.org/english/ res_e/reser_e/ersd201306_e.pdf 